\title{
palacio de correos, telégrafos y oficinas públicas en cortina d'ampezzo
}

EDOARDO GELLNER, arquitecto, EDMONDO y EDGARDO PROTTI, ingenieros

$145-60$

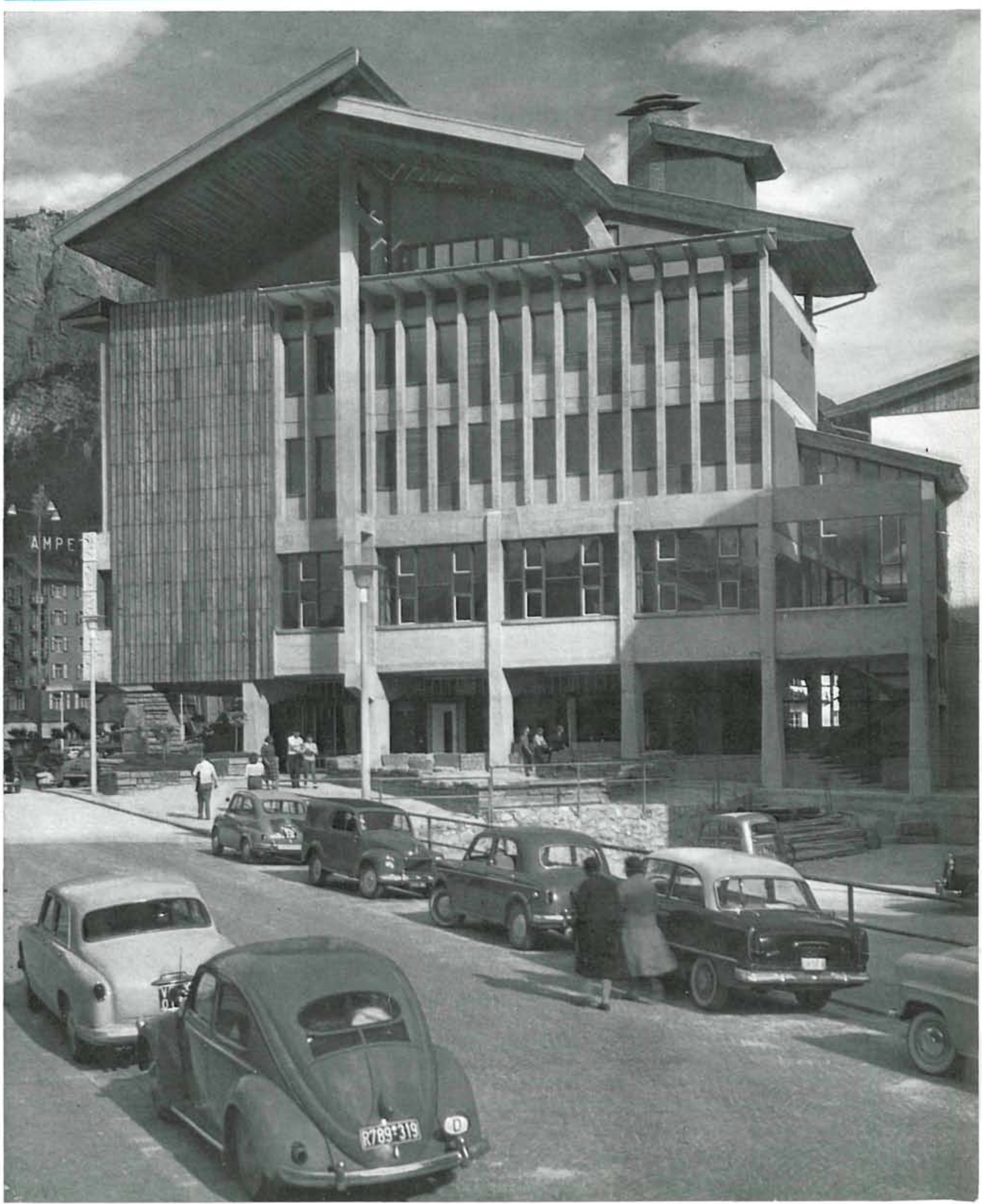




\section{simopsis}

E1 edificio aloja en el semisótano los locales de ingreso y maniobra con acceso cubierto para automóviles, garaje, archivo, ropero, aseos del personal y calderas de calefacción.

La planta baja alberga: salón para el público, escritorio, servicios de imposición de telegramas, paquetes, apartado de correos y oficinas generales. Estos dos pisos se comunican mediante escaleras y montacargas.

El primer piso está ocupado por los servicios de telecomunicación, sala de teletipos, almacenes, grupo electrógeno, y vestuario y aseos para el personal auxiliar. En la otra parte de este piso, disponiendo de accesos independientes, está ubicada la sala de Audiencias del
Juzgado.

Se desarrollan en el segundo piso las dependencias del Juzgado: oficinas del juez, de los secreta: dística, archivo $\mathrm{y}$ registro.

En el tercer piso están las oficinas de Hacienda y seguridad públicas. $Y$ en el ático, los salones del círculo artistico, capaces para 120 personas, eon bar, oficinas $y$

E1 edificio ofrece toda su estructura de hormigón armado vista y abujardada. La policromía de tados de color azul celeste y piny los revestimientos de y rojo, $y$ los revestimientos de alerce, tribuyen a romper el tono gris uniforme de la masa de hormigón armado y dan vivacidad a las fachadas.

\section{detalle}

\section{fachada oeste}

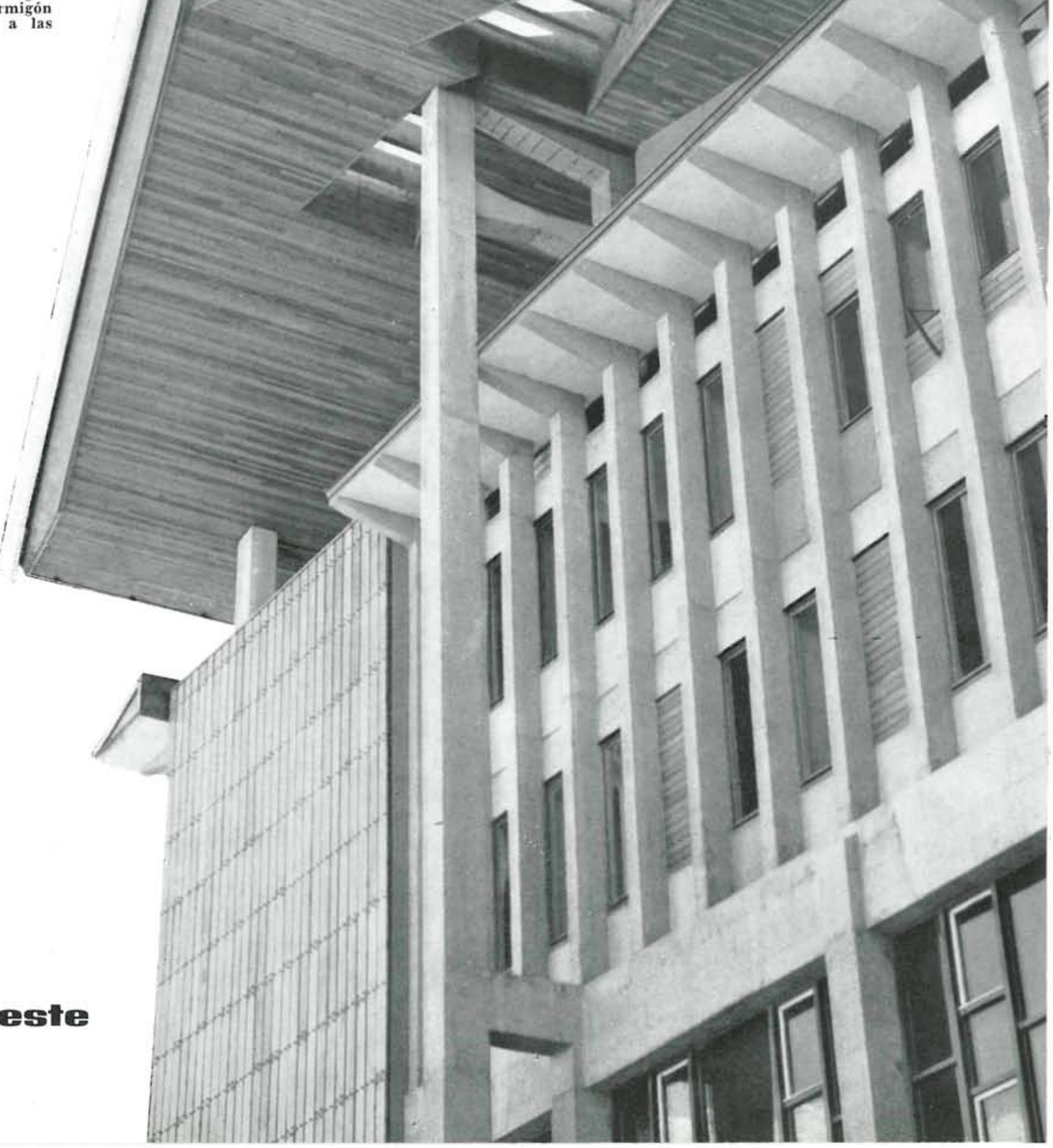




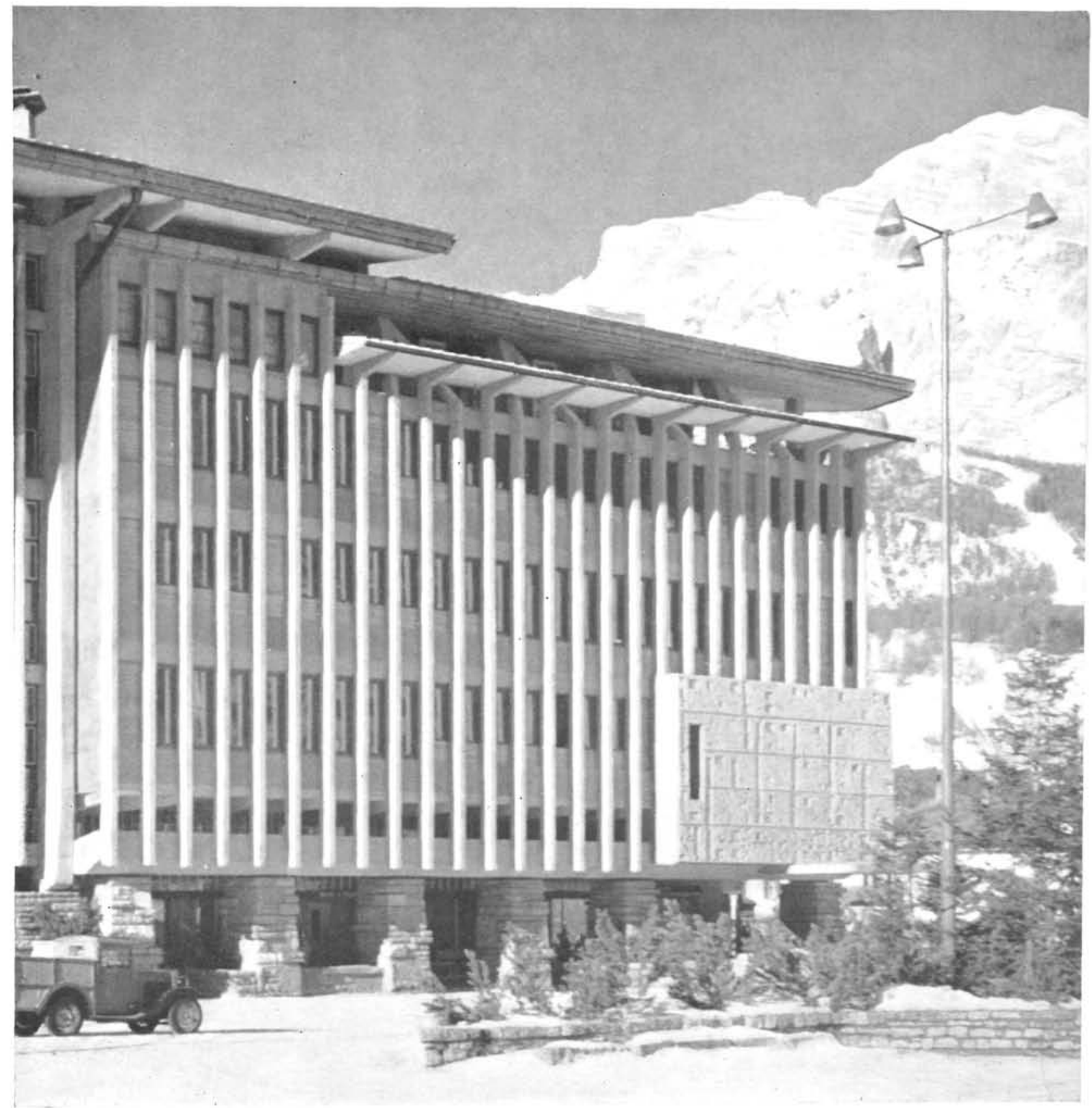

Destino mixto.-El fin de este edificio es dar cabida a los servicios de:

a) Correos y telégrafos;

b) Oficinas públicas: Juzgado, Registro, Hacienda y Seguridad;

c) Sala de reunión del Círculo Artístico (entidad cultural).

Distribución.-Los servicios de correos y telégrafos ocupan parcialmente los tres pisos bajos del edificio; los dos más altos están reservados para oficinas públicas, y el Círculo Artístico aparece instalado en el ático.

El semisótano aloja: locales de ingreso y maniobra con acceso cubierto para automóviles, garaje, archivo, vestuario y aseos del personal y central de calefacción.

En planta baja están los servicios postales: sala para el público, escritorio y venta de periódicos, servicios de imposición de giros, de telegramas, paquetes, apartado de correos y oficinas generales. Las dos plantas mencionadas se comunican por medio de escaleras y montacargas. 

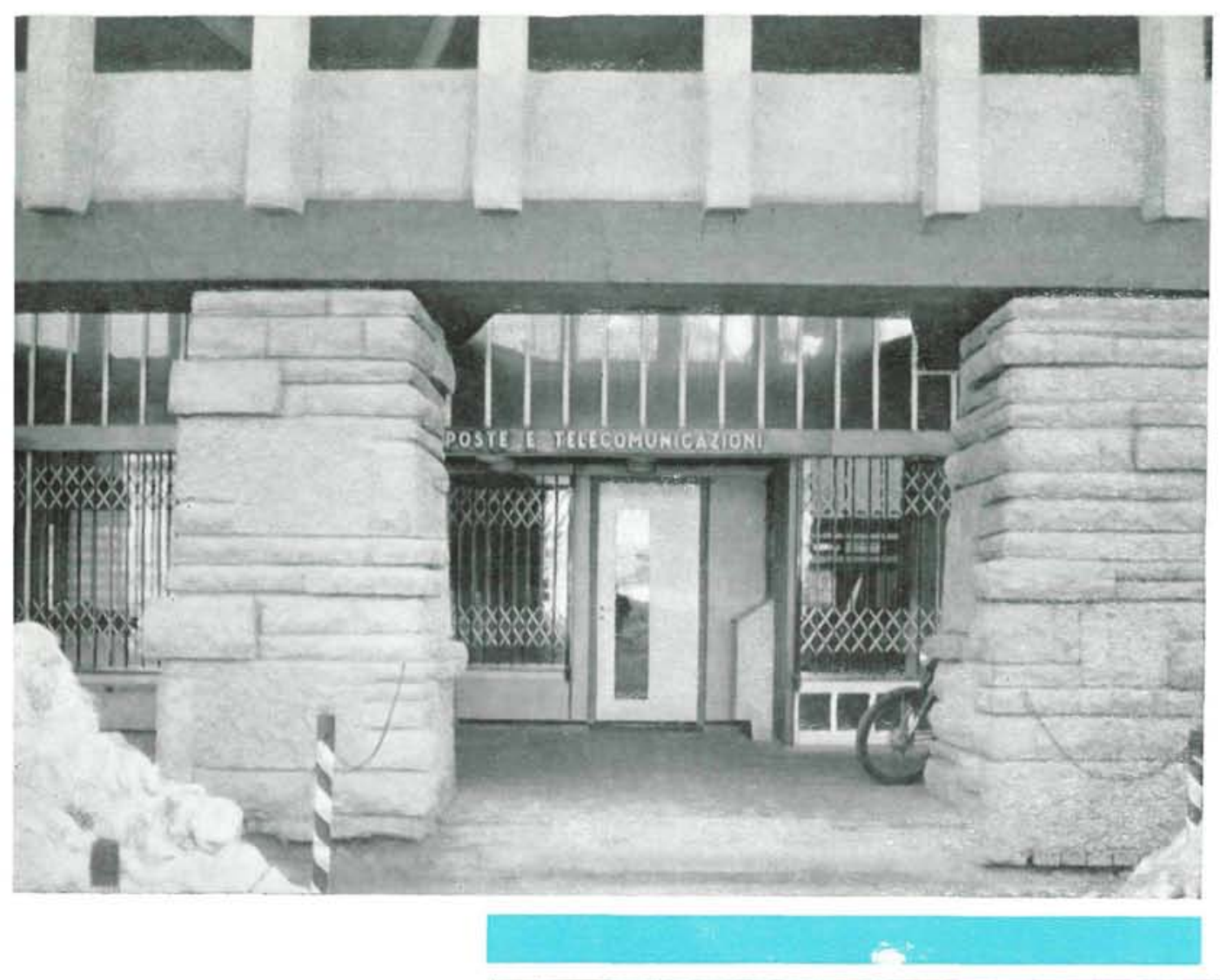

En el primer piso se disponen los servicios de telecomunicación: salas de teletipos, almacenes, grupo electrógeno, vestuario y aseos para ei vestuario y aseos para el personal. En otra parte de este piso, con acce-
sos independientes, está situada la sala de Audiencias del Juzgado.

En el segundo piso se distribuyen las dependencias del Juzgado: despacho del juez, de los secretarios $10^{\circ}$ y $2 .^{\circ}$, de los secretarios $10^{\circ}$ y $2 .^{\circ}$,
sala de abogados-unida por una escalera de caracol con la cie Audiencia-, oficinas, estadística, archivo y registro.

Tercer piso. - Lo ocupan las oficinas de Hacienda y Seguridad.

Atico.-En él están instalados: un salón de reunión del Círculo Artístico-capaz para 120 personas-, bar, oficinas y aseos; el salón tiene comunicación con amplias terra. zas, $\mathrm{y}$ un ascensor une directamente el ático con la entrada a la planta baja.

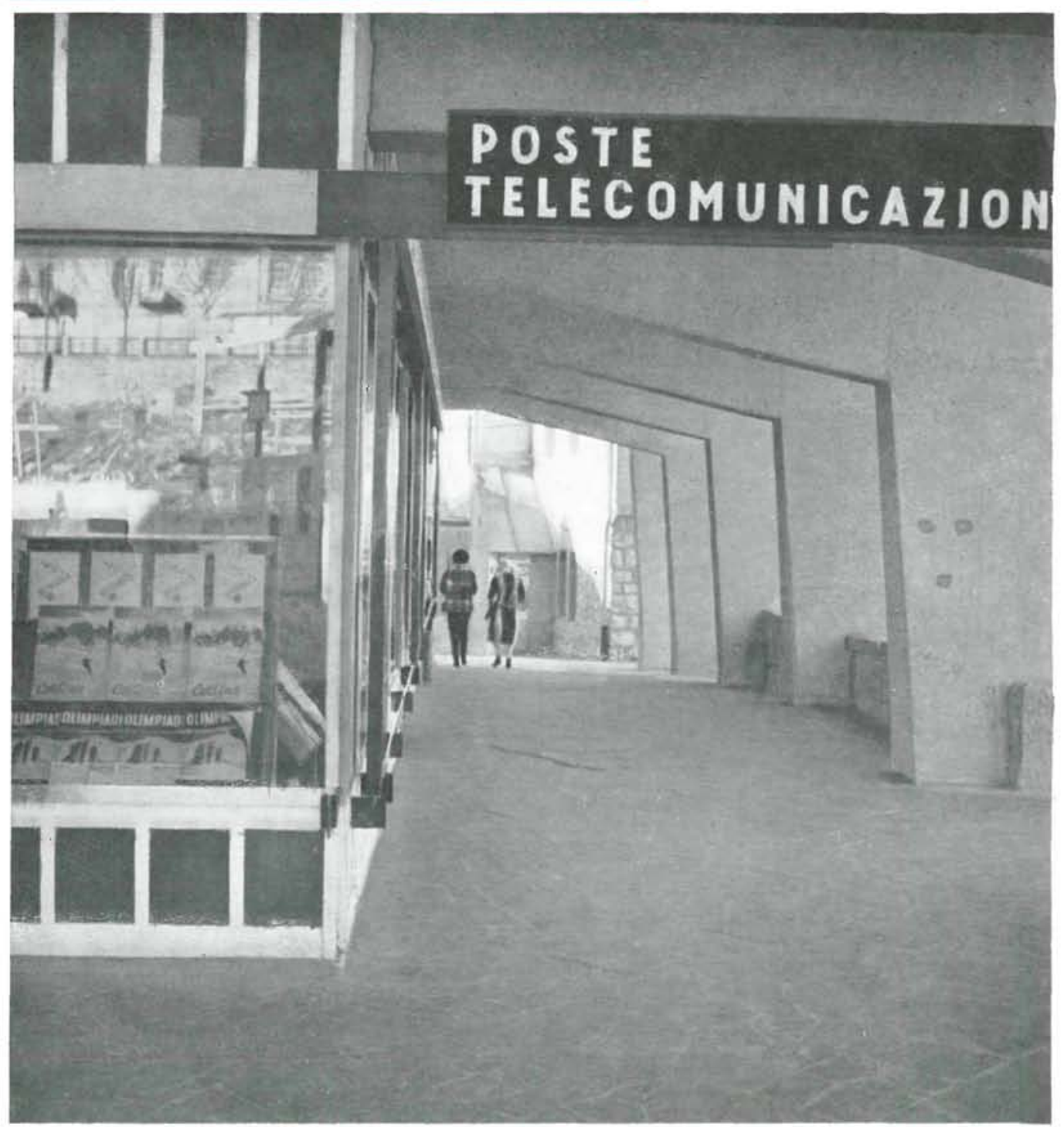




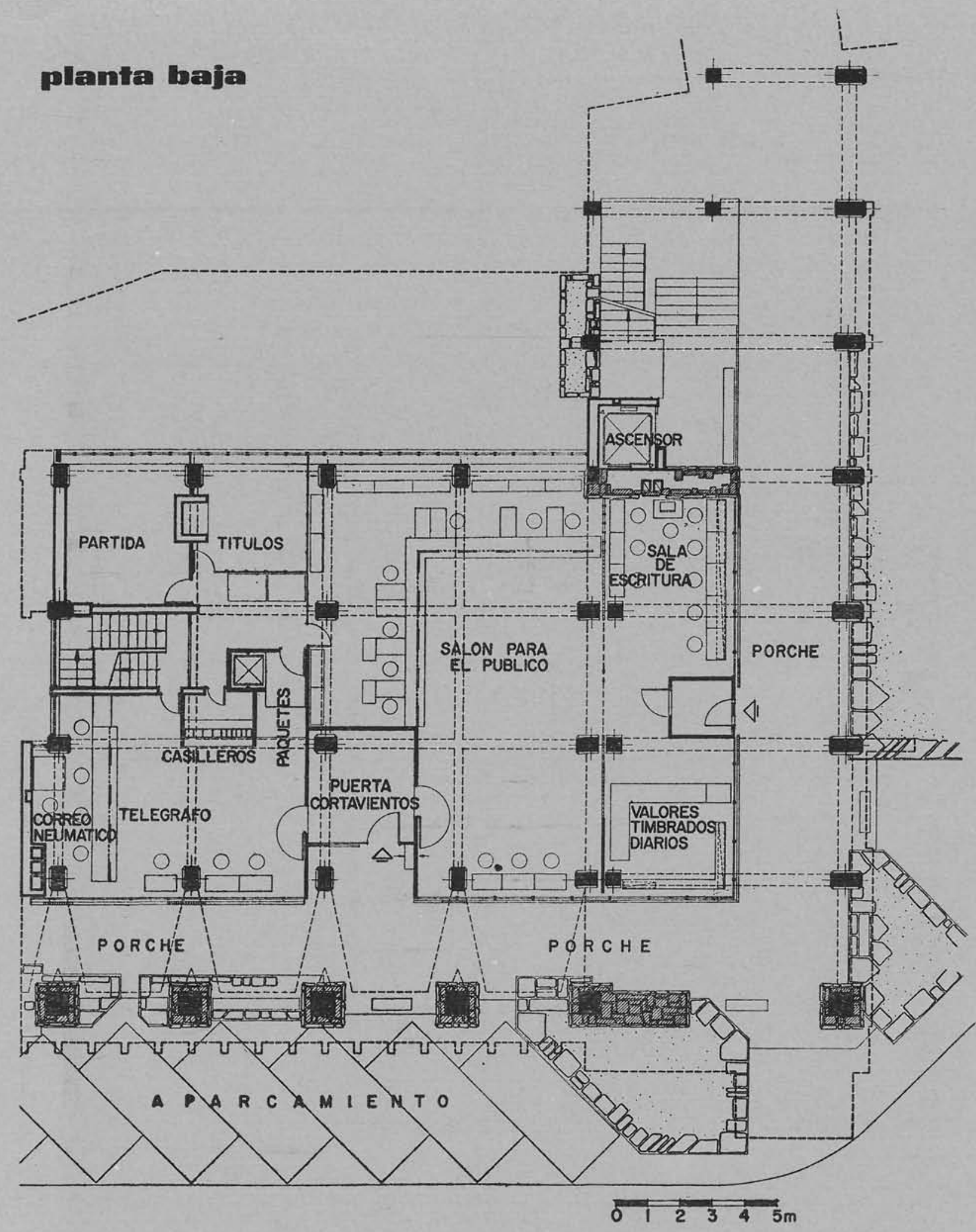

Estructura.-Es resistente, de hormigón armado, y presenta una inversión-en planta baja-de la dirección del entramado principal, acusada por el perfll peculiar que ad optan los pilares como puede verse en la sala para el público.

Por el contrario, la estructura sustentante de la fachada está constituida por una serie de delgadas pilastras que transmiten su carga a otras, en número menor, de la planta baja, mediante una gruesa viga horizontal. Las pilastras de fachadas se unen en horquilla-en el ático-formando las armaduras de la cubierta.

Los cerramientos verticales son de fábrica de ladrillo con doble capa de aislante Eraclit y enfoscado o revestimiento de madera como capa final. 


\section{planta primera}

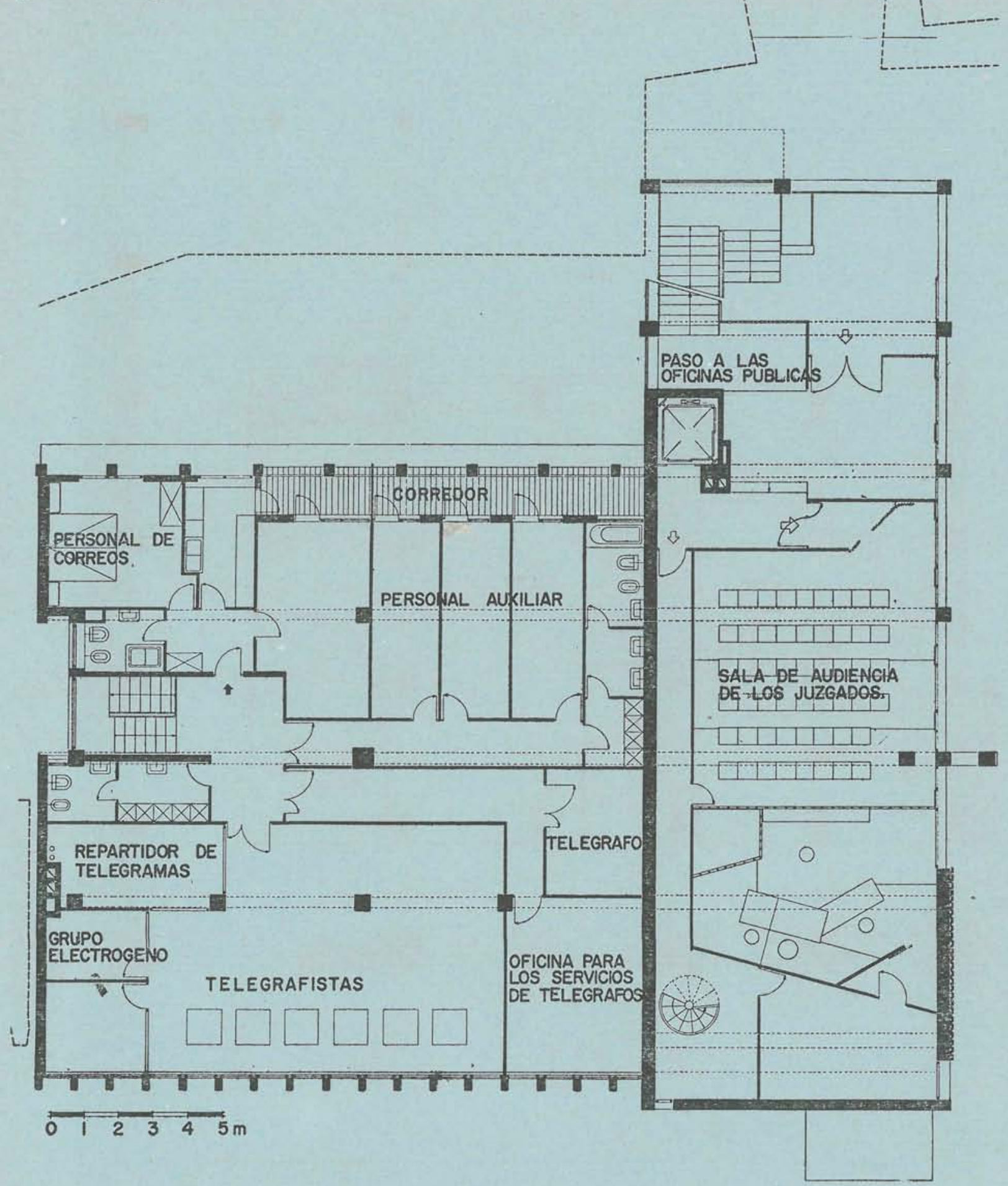

Criterios arquitectónicos.-El edificio ofrece su estructura de hormigón armado "vista y abujardada". La policromia de los paneles de cerramiento, pintados de color azul celeste y rojo, y los revestimientos de alerce, en su color natural rojizo, contribuyen a romper el tono gris uniforme de la masa de hormigón armado y dan veracidad a las fachadas.

Respecto a la cubierta, se ha tratado de formar aparentemente pesada en concordancia con el edificio, y presenta un fuerte vuelo hacia la fachada noroeste, estando parcialmente sostenido por un pie derecho que alcanza toda la altura de la fachada. 
planta segunda
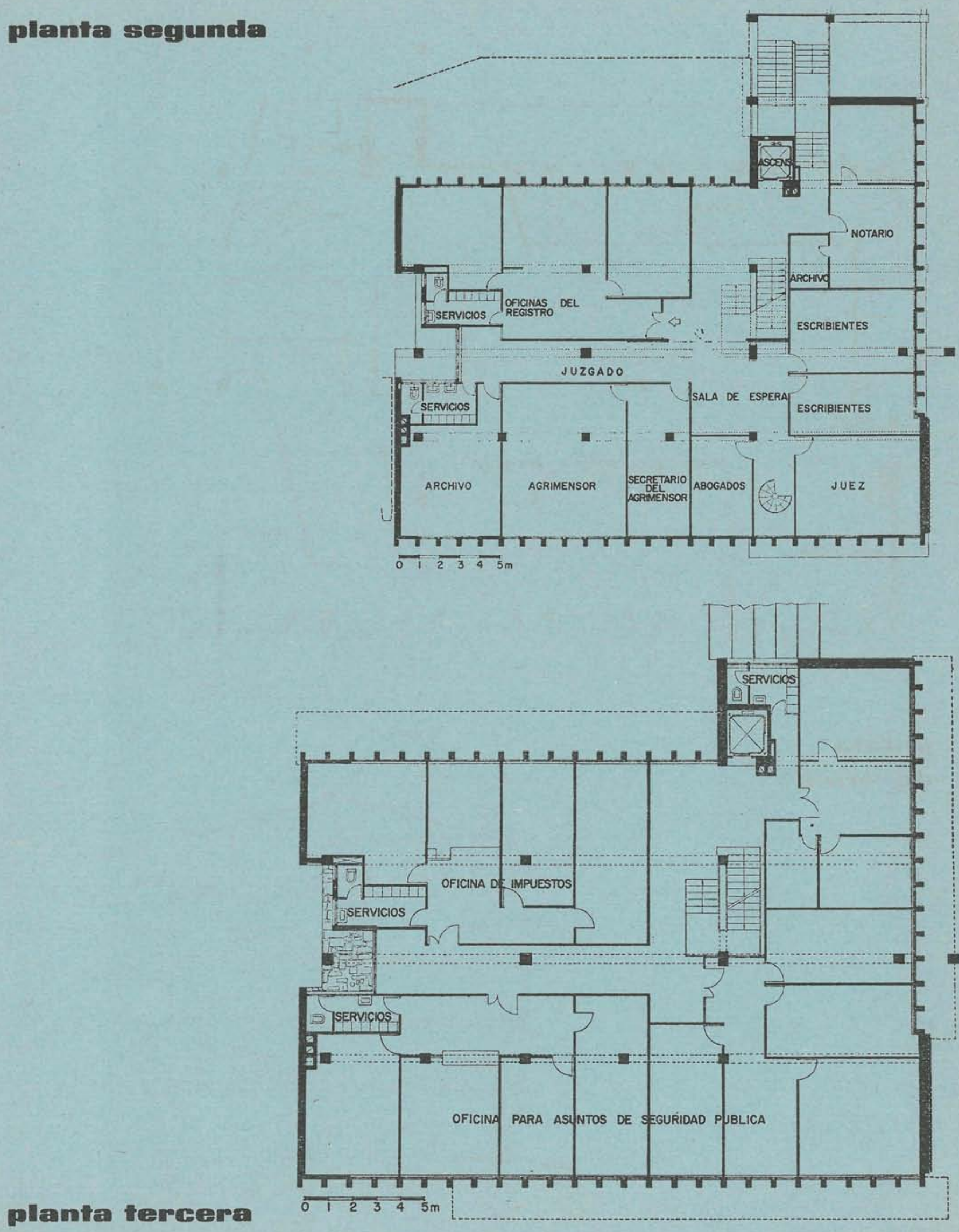


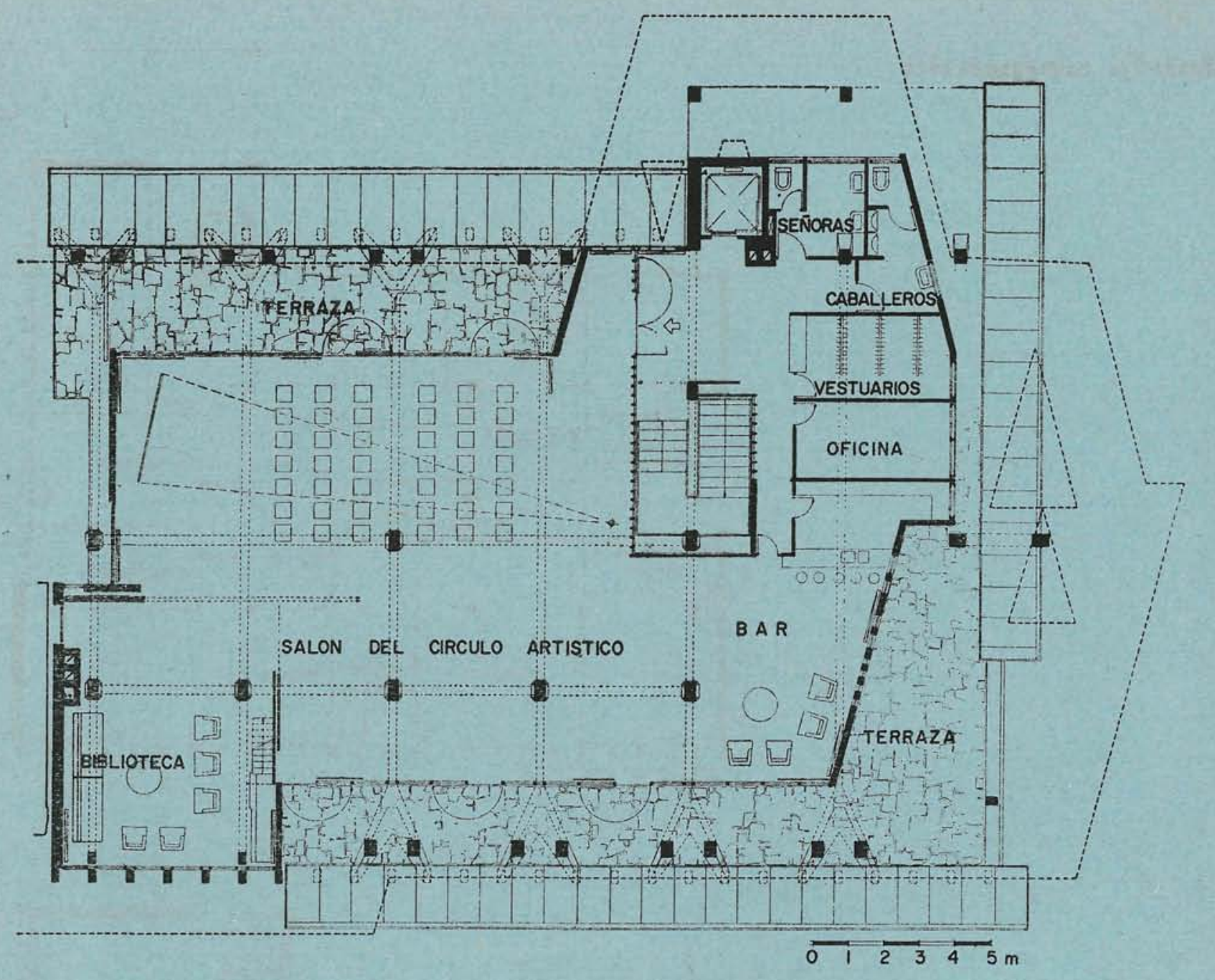

\section{plante \\ de ático}

\section{SEcceín}

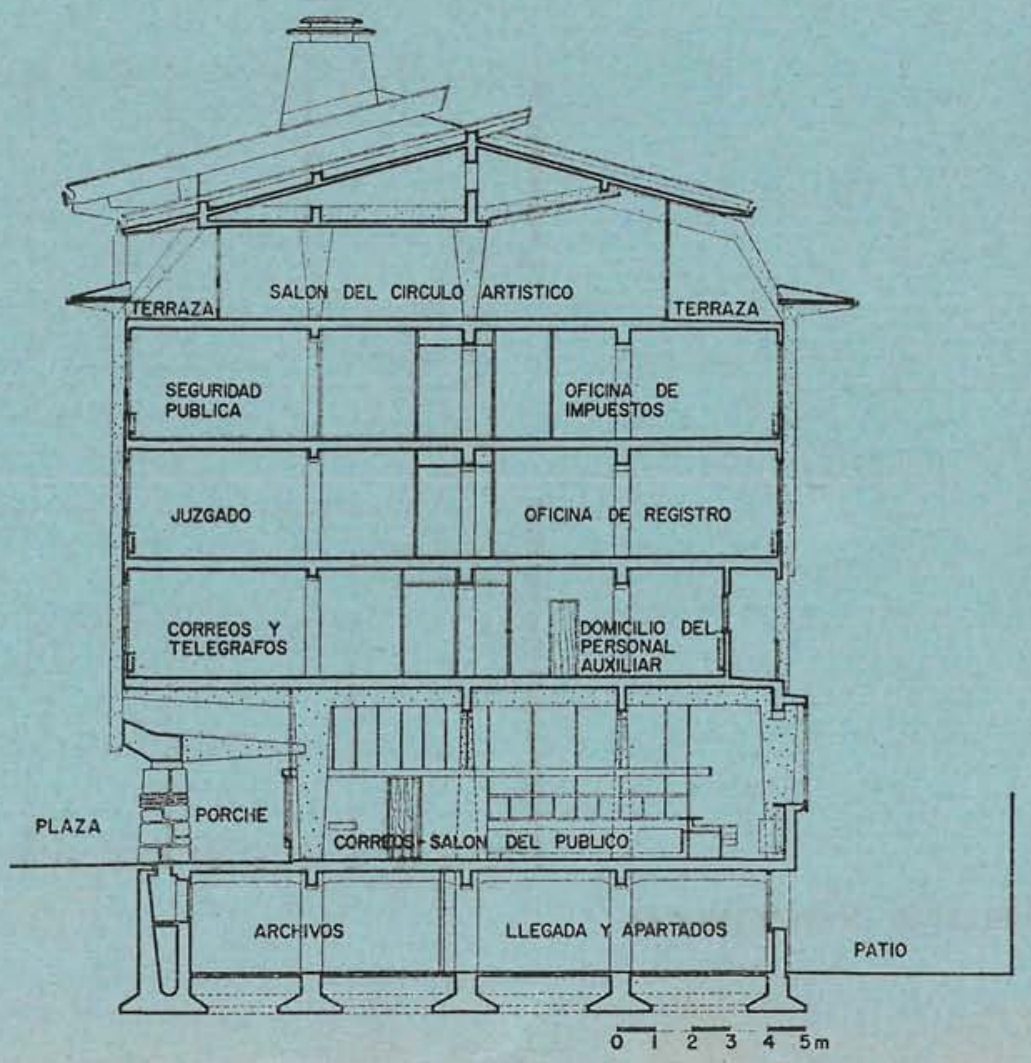




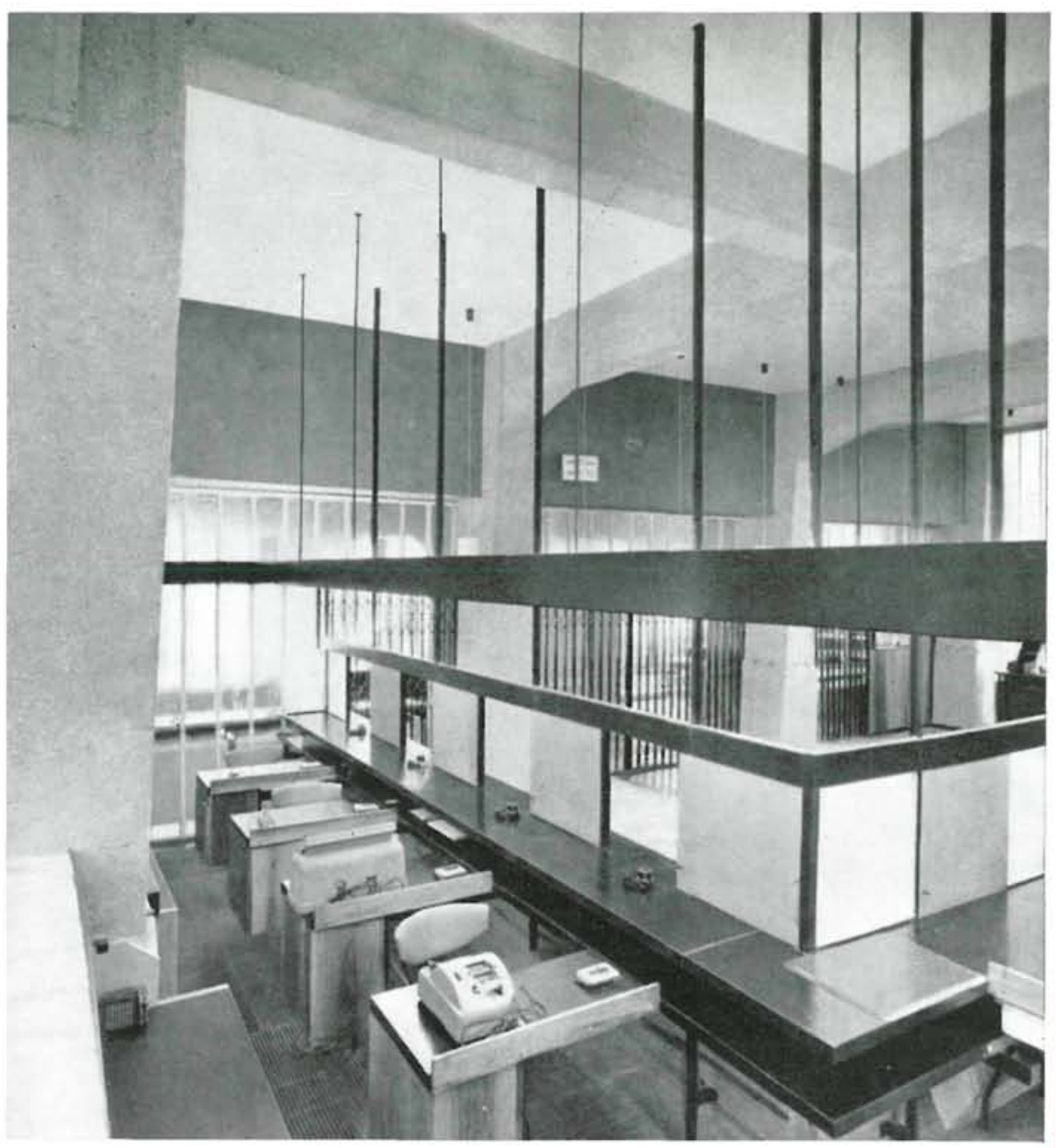

Dando un mayor realce a la cubierta a dos aguas de esta fachada, que es la única visible desde el antiguo Centro de Cortina, se ha querido establecer una relación, con la edificación tradicional de montaña, caracterizada precisamente por tal tipo de cubierta, todavía predominante en el viejo ambiente.

Los soportales que circundan la construcción, por los dos lados que dan a la plaza, tienen unas proporciones similares a las del edificio vecino - "Telve"-que será tratado en otro artículo Se ha proyectado a "escala humana", lo que le imprime ese atractivo del que gozan los típicos soportales de las ciudades venetas y del Alto Adigio.

El voladizo de hormigón armado -que cubre la zona porticada-está tratado con cemento coloreado azul celeste; los revestimientos de la planta baja están realizados con piedra roja de Castellavazzo y el pavimento de los soportales se compone de toscas baldosas irregulares de pórfido.
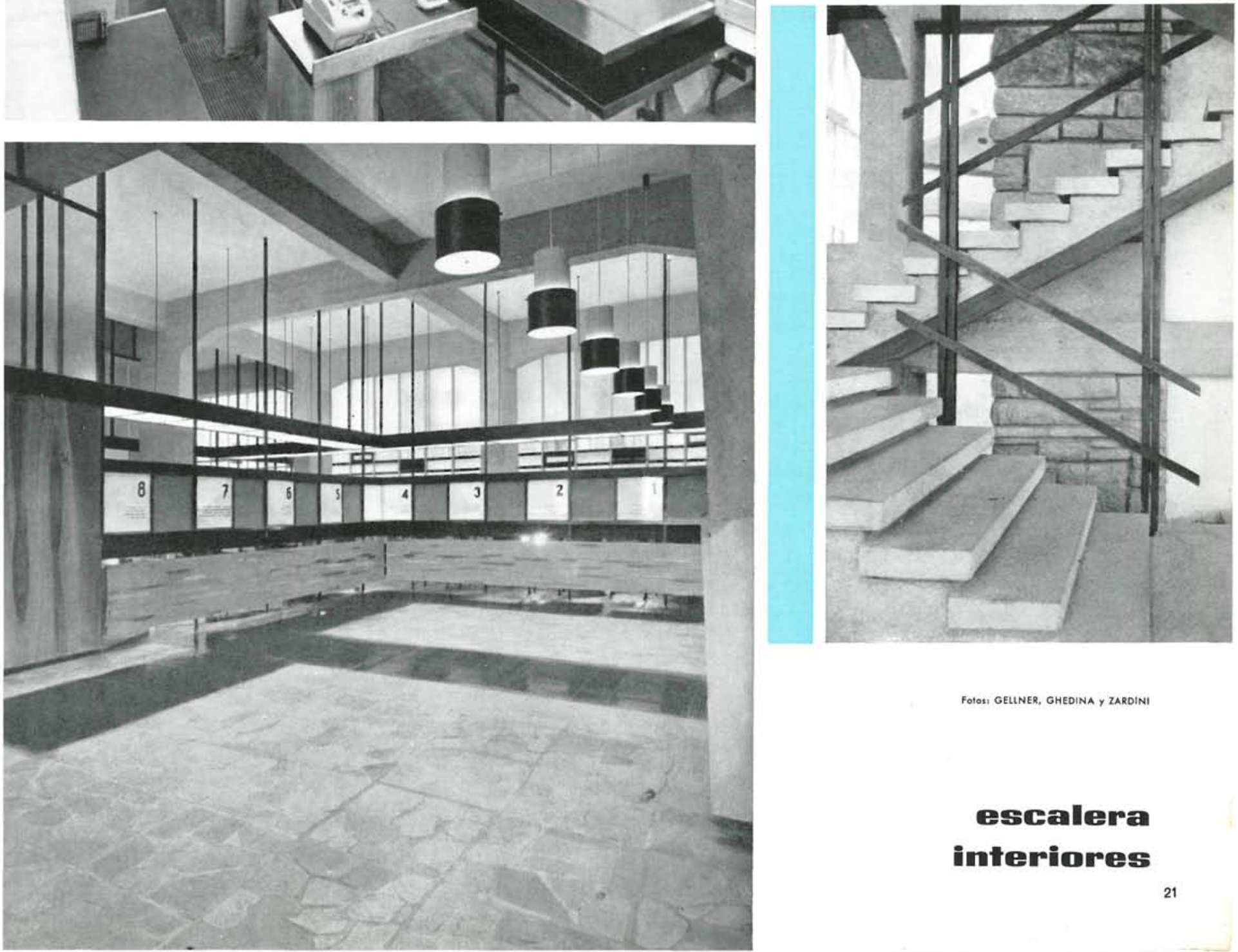

Folo: GELINER, GHEDINA Y ZARDINI

escealema interiores 


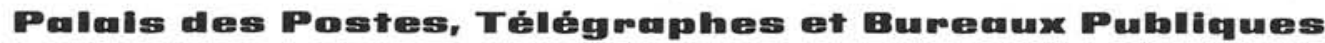 ci Corrtima d'Ampezzo}

Edoardo Gellner, architecte.

L'édifice abrite au sous-sol les locaux d'entrée et de manoeuvre avec accès couvert pour voitures, garage, archives, vestiaires, toilette pour le personnel et les chaudières de chauffage.

Au rez-de-chaussée se trouvent le salon pour le public, un bureau, les services de télégrammes, colis, poste restante et les bureaux généraux. Ces deux étages communiquent entre eux par des escaliers et des monte-charge.

Le premier étage est occupé par les services de télécommunication, la salle de télétypes, les magasins, le groupe électrogène, le vestiaire et la toilette pour le personnel auxiliaire. Dans l'autre partie de cet étage et disposant d'accès indépendants, se trouve la salle d'Audiences du Tribunal.

Au deuxième étage sont situés les locaux du Tribunal: le cabinet du juge, les bureaux des secrétaires, la salle des avocats, la salle de statistique, les archives et le bureau d'enregistrement.

Au troisième étage se trouvent les bureaux de Finances et de Sécurité Publique. Dans l'attique, les salons du cercle artistique, capables d'abriter 120 personnes, avec un bar, des bureaux et des toilettes.

L'édifice montre toute sa structure, en béton armé, vue et bouchardée. La polychromie des panneaux de fermeture, peints en bleu ciel et rouge, et les revêtements en mélèze, avce leur couleur naturelle rougeâtre, contribuent à rompre le ton gris
uniforme de la masse de béton armé et donnent de la vivacité aux façades.

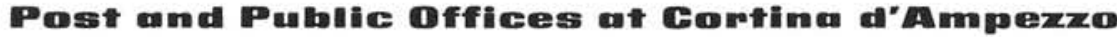

Edoardo Gellner, architect.

The basement of this building houses the arrival and distribution departments, and there is a covered entry for motocars. Room is also found in the basement for a garage, the archives, the dressing rooms, washrooms, and heating boilers.

The ground floor includes: hall for the public, writing room, telegram despateh department, parcel post section, post restante and general offices. The ground floor and the basement are connected by stairs and lifts.

The first floor accommodates the telegraphing department, teletypes, stores, private diesel power plant, and dressing and washing rooms for the staff. Another part of this floor, with separate access, is taken up with the Court offices.

The second floor houses other court ancillaries, including the judge's office, and that of the secretaries, the lawyer's room, the statistics department, the archives and the registry office.

The Treasury and Public Security Offices are located on the third floor. The attic provides room for the artistic circle rooms, which can accommodate 120 people, and includes a bar, offices and washrooms.

The reinforced concrete structure of the building is externally visible. Multicoloured panels fill the conerete framework are painted in sky blue and red and greatly help to break up the uniform grey tone of the reinforced concrete mass. The contrast between the two patterns also enhances the geometric quality of the total design.

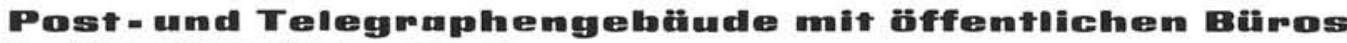

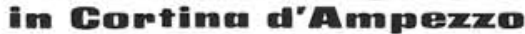

Edoardo Gellner, Architekt.

Im Tiefparterre des Gebäudes sind Ankunfts- und Arbeitshallen mit einem überdachten Zugang für Fahrzeuge, Garrage, Archiv, Umkleideräume und Waschräume für das Personal und die Heizungskessel untergebracht.

Im Parterre befinden sich: Halle für den Publikumsverkehr, Schreibraum, Telegrammaufgabe, Paketaufgabe, Schliessfächer und Büros.

Die beiden eben beschriebenen Stockwerke sind durch Treppen und Lastenaufzüge untereinander verbunden.

Im ersten Stockwerk sind Fernsprech-und Fernschreibanlage, Lager, Stromerzeuger und Umkleide- und Waschräume für das Hilfspersonal untergebracht. Im anderen Teil desselben Stockwerkes liegt der Gerichtsaal.

Im zweiten Stockwerk befinden sich die einzelnen Räume des Gerichtes: die Büroräume des Richters, der Sekretäre und Rechtsanwälte und die Räume für Statistik, Archiv und Register.

Im dritten Stockwerk liegen die Räume des Finanzamtes und der Öffentlichen Sicherheit. Im letzten Stock befinden sich die Räume des Künstlerkreises mit einer Kapazität von 120 Personen, Erfrischungsraum, Büros und Waschräume.

Die Struktur des Gebäudes besteht aus bearbeitetem Sichtstahlbeton. Die Farbenfreudigkeit der Umfassungsmauern, die in himmerlblau und rot gehalten sind und die Lärchenholzverkleidung mit ihrer rötlichen Naturfarbe tragen dazu bei, das cinförmige Grau des Stahlbetons zu unterbrechen und Leben in die Fassade zu bringen. 